BULL. AUSTRAL. MATH. SOC.

VOL. 32 (1985), 55-68.

\title{
ON THE PROPERTIES OF AiN INTEGRAL OPERATOR
}

\section{ZBIGNIEW JERZY JAKUBOWSKI}

\begin{abstract}
The main aim of the paper is to examine the properties of the integral operator $I(f)=\int_{0}^{z}\left[f^{\prime}(u)\right]^{\alpha} d u, \alpha \in C$, defined on some classes of functions $f(z)=z+a_{2} z^{2}+\ldots$ univalent and convex in the disc $|z|<1$. As special cases we obtain results of Kim and Merkes and of Kumar and Shukla.
\end{abstract}

1.

Let $K\left(z_{0}, r\right)=\left\{z \in \mathbb{C}:\left|z-z_{0}\right|<r\right\}, r>0, K=K(0, I)$, Let $U$ be any fixed simply connected domain contained in the half-plane $\operatorname{Re} w>0$ and containing the point $w=1, G=G\left(\xi, n_{0}, n_{1}, \ldots, \eta_{n}\right)$ - a given function defined in a sufficiently large domain $w \subset \mathbb{C}^{n+2}$, with $G\left(0,0,1, \eta_{2}, \ldots, \eta_{n}\right)=1$. Denote by $F(G, U)$ the class of functions

$$
f(z)=z+a_{2} z^{2}+\cdots
$$

holomorphic in $K$ and satisfying in this disc the condition

$$
G\left(z, f(z), f^{\prime}(z), \ldots, f^{(n)}(z)\right) \in U
$$

It is evident that, with the right specification of the domain $U$ and the

Received 7 December 1984.

Copyright Clearance Centre, Inc. Serial-fee code: 0004-9727/85 $\$ A 2.00+0.00$. 
function $G$, we obtain from (2) the definitions of various well-known classes of functions of type (I).

Let $F^{*}(G, U)$ be a subclass of functions of the family $F(G, U)$, locally univalent in $K$. Then, for each function $f \in F^{*}(G, U)$, $f^{\prime}(z) \neq 0$ in the disc $K$. Consequently, for any complex $\alpha$, on the family $F^{*}=F^{*}(G, U)$ one can define an integral operator of the form

$$
I(f)=\int_{0}^{z}\left[f^{\prime}(u)\right]^{\alpha} d u, 1^{\alpha}=1 .
$$

of course: in the case $\alpha=1$, the operator $I$ maps $F^{*}$ onto $F^{*}$; when $\alpha=0, I\left(F^{*}\right)=z$. Certain questions concerning the properties of operator (3) seem natural. In particular: whether or not, and for which $\alpha \in \mathbb{C}, I\left(F^{*}\left(G, U_{1}\right)\right)=I\left(F^{*}\left(G, U_{2}\right)\right)$.

In $1974 \mathrm{Kim}$ and Merkes [8] showed that, for each $0 \leq \alpha \leq 1$, if (1) is any convex univalent function, then $I(f)$ is also a convex function; this interval cannot be enlarged in the class of all convex functions.

In the present paper, we consider the properties of operator (3) in some classes of convex functions. As special cases we obtain the abovementioned result [8] and those of Kumar and Shukla [10]. We also give some results concerning the extimations of coefficients.

\section{2.}

Let $E=E_{1} \cup E_{2}$ where

$$
E_{1}=\left\{(s, \rho) \in \mathbb{C} \times \mathbf{R}:|s-1|<\rho, 0<\rho \leq \frac{1}{2}\right\} \text {, }
$$

$$
E_{2}=\left\{(s, \rho) \in \mathbb{C} \times \mathbb{R}:|s-1|<\rho \leq \operatorname{Re} s, \rho>\frac{1}{2}\right\} .
$$

Denote by $S^{c}(s, \rho),(s, \rho) \in E$ (see [4]), the class of functions of form (I) holomorphic in $K$ and satisfying the condition

$$
1+z f^{\prime \prime}(z) / f^{\prime}(z) \in K(s, \rho), z \in K \text {. }
$$

From (4) we infer that if $w \in K(s, \rho)$, then $\operatorname{Re} w>\operatorname{Re} s-\rho \geq 0$. Consequently, $S^{c}(s, \rho) \subset S^{c}$ where $S^{c}$ is the well-known family of functions of the form (1) univalent and convex in the disc $K$. 
Since the homography

$$
h(z)=(1+a z) /(1+b z), z \in K,
$$

where

$$
\text { (7) } \begin{aligned}
a=\left(\rho^{2} e^{i \gamma}+s|s-1|\right) \rho^{-1}, b=|s-1| \rho^{-1}<1 ; & \gamma=\arg (1-s), \\
& s \neq 1 ; \gamma=0, s=1,
\end{aligned}
$$

maps the disc $K$ onto $K(s, \rho)$, therefore condition (5) is equivalent to

$$
1+z f^{\prime \prime}(z) / f^{\prime}(z) \prec h(z), z \in K,
$$

(the symbol $\prec$ stands for subordination). In papers [4], [6], the class $S^{c}(s, \rho)$ was considered when $(s, \rho) \in E, s=\bar{s}$. Also, other classes of functions (starlike, Carathéodory and the like) satisfying the condition of type $G\left(z, f(z), f^{\prime}(z), f^{\prime \prime}(z)\right) \in K(s, \rho)$ were investigated when $(s, p) \in E$ (for example, in [1], [5], [11]) or when $(s, \rho) \in E, s=\bar{s}$ (for example, in [1] to [3], [5] to [7], [9], [11]).

Let $f \in S^{c}(s, p),(s, \rho) \in E, \alpha \in \mathrm{C}$ and

$$
F=I(f)
$$

Then, from (3), we have

$$
E^{\prime}(z)=\left(f^{\prime}(z)\right)^{\alpha}, z \in K
$$

Consequently,

$$
1+z F^{\prime \prime}(z) / F^{\prime}(z)-\sigma=\alpha\left[1+\left(z f^{\prime \prime}(z) / f^{\prime}(z)\right)-s\right], z \in K,
$$

where

$$
\sigma=1-\alpha+\alpha s .
$$

So, from (11), we get

$$
1+z F^{\prime \prime}(z) / F^{\prime}(z) \in K(\sigma, R), z \in K,
$$

where

$$
R=|\alpha| \rho,
$$

while $\sigma$ is defined by formula (12).

Since $(s, p) \in E$, therefore, for each $\alpha \in \mathbb{C}$, from (4), (12) and (14) we have $|\sigma-1|=|\alpha s-\alpha|<|\alpha| \rho=R$; so, for each $\alpha \in \mathbb{C}$, 
In consequence, $K(\sigma, R) \cap K(s, \rho) \neq \varnothing$.

\section{3.}

Let us consider, for any fixed $(s, \rho) \in E$, the following sets of points $\alpha \in \mathbb{C}$ :

$$
\begin{aligned}
& D_{1}=D_{1}(s, \rho)=\{\alpha:|s-1||\alpha-1|+\rho|\alpha| \leq \rho\}, \\
& D_{2}=D_{2}(s, \rho)=\{\alpha: \cdot|s-1||\alpha-1|+\rho \leq|\alpha| \rho\}, \\
& D_{3}=D_{3}(s, \rho)=\{\alpha:|\alpha| \rho \leq \operatorname{Re}(1-\alpha+\alpha s)\}, \\
& D_{4}=D_{4}(s, \rho)=\mathbb{C} \backslash\left(D_{1} \cup D_{2}\right) .
\end{aligned}
$$

Evidently :

$I^{\circ} \quad D_{1}(s, \rho) \subset D_{1}(1, \rho)=\{\alpha:|\alpha| \leq 1\} ;$

$2^{\circ}$ the points $0,1, \alpha_{1} \in D_{1}$ where $\alpha_{1}=-(\rho-|s-1|)(\rho+|s-1|)^{-1}$, $-1 \leq \alpha_{1}<0$

$3^{\circ} \alpha \in D_{1}$ if and only if $\bar{\alpha} \in D_{1}$;

$4^{\circ}$ the boundary of the set $D_{1}$, in polar coordinates, is defined by the equation

(20) $r=r_{1}(t)$

$$
\begin{array}{r}
\equiv\left(1-b^{2}\right)^{-1}\left[1-b^{2} \cos t-\left(2 b^{2}(1-\cos t)-b^{4}\left(1-\cos ^{2} t\right)\right)^{\frac{1}{2}}\right], \\
0 \leq t \leq 2 \pi,
\end{array}
$$

with $r_{1}^{\prime}(t)<0$ for $0<t<\pi$ and $r_{1}^{\prime}(t)>0$ for $\pi<t<2 \pi$, and $b$ is defined by formula $(7)$.

Moreover :

$1^{\circ} D_{2}(s, \rho) \subset D_{2}(1, \rho)=\{\alpha:|\alpha| \geq 1\} ;$

$2^{\circ}$ the points $1,1 / \alpha_{1} \in D_{2}$;

$3^{\circ} \quad \alpha \in D_{2}$ if and only if $1 / \alpha \in D_{1}, \alpha \neq 0$; 
$4^{\circ}$ the boundary of the set $D_{2}$ is defined by the equation $r=r_{2}(t), 0 \leq t \leq 2 \pi$, where $r_{2}(t)=r_{1}^{-1}(t)$, and $r_{1}(t)$ is defined by $(20)$.

It is also evident that:

$1^{\circ} D_{3}(1, \rho)=\left\{\alpha:|\alpha| \leq \rho^{-1}\right\} ;$

$2^{\circ}$ the points $0, I, \alpha_{2}, \alpha_{3} \in D_{3}$ where $\alpha_{2}=(\rho+I-\operatorname{Re} s)^{-1}$, $\alpha_{3}=(1-\rho-\operatorname{Re} s)^{-1}$ with $\alpha_{2} \geq 1, \alpha_{3}<-(2 \rho)^{-1}$;

$3^{\circ}$ the boundary of the set $D_{3}$ is defined by the equation $r=r_{3}(t) \equiv \rho^{-1}[1+\tau \cos (t+\gamma)]^{-1}, 0 \leq t \leq 2 \pi$, where
$\tau \rho e^{i \gamma}=1-s, 0 \leq \tau<1,0 \leq \gamma<2 \pi ;$ moreover, we have
$\alpha_{4} \leq r_{3}(t) \leq \alpha_{5}$ where $\alpha_{4}=(\rho+|s-1|)^{-1}, \alpha_{5}=(\rho-|s-1|)^{-1}$. So, from (16-19) we get:

$1^{\circ} D_{1}(s, \rho) \cap D_{2}(s, \rho)=\{1\}$ when $s \neq 1$, and $D_{1}(I, \rho) \cap D_{2}(1, \rho)=\{z:|z|=1\}$;

$2^{\circ} \quad D_{4}(1, \rho)=\varnothing ;$

$3^{\circ} D_{2}(1, \rho) \cap D_{3}(1, \rho)=\left\{\alpha: 1 \leq|\alpha| \leq \rho^{-1}\right\}$

$4^{\circ} D_{2}(s, \rho) \cap D_{3}(s, \rho) \neq \emptyset$ when $s \neq 1$.

Let us also notice that

$$
D_{1} \subset D_{3}
$$

Indeed, if there existed points $\left(s_{0}, p_{0}\right) \in E$ and $\alpha_{0} \in D_{1}$ such that $\alpha_{0} \notin D_{3}$, then we would have, from (18),

$$
\left|\alpha_{0}\right| \rho_{0}>\operatorname{Re}\left[\left(\alpha_{0}-1\right)\left(s_{0}-1\right)\right]+\operatorname{Re} s_{0} .
$$

Since $a_{0} \in D_{1}$, therefore from (16) there would be 


$$
\rho_{0}>\operatorname{Re}\left[\left(\alpha_{0}-1\right)\left(s_{0}-1\right)\right]+\left|\alpha_{0}-1\right|\left|s_{0}-1\right|+\operatorname{Re} s_{0} .
$$

This last inequality is a contradiction since from (4) it follows that $\operatorname{Re} s_{0}-\rho_{0} \geq 0$.

So, from (12), (14), (15) and (16-19) we get, for any $(s, \rho) \in E$,

$$
\begin{aligned}
& K(\sigma, R) \subset K(s, \rho) \Leftrightarrow \alpha \in D_{1}, \\
& K(s, \rho) \subset K(\sigma, R) \Leftrightarrow \alpha \in D_{2}, \\
& K(\sigma, R) \subset T=\{w: \operatorname{Re} w>0\} \Leftrightarrow \alpha \in D_{3},
\end{aligned}
$$

(25) $K(\sigma, R) \backslash K(s, \rho) \neq \varnothing$ and

$$
K(s, \rho) \backslash K(\sigma, R) \neq \emptyset \Longleftrightarrow \alpha \in D_{4},(1 \in K(\sigma, R) \cap K(s, \rho)) \text {. }
$$

As a consequence we obtain, from (21-25).

LEMMA. Let $(s, \rho) \in E, \alpha \in \mathbb{C}$. Then

$$
\begin{array}{ll}
\text { (26) } K(\sigma, R)=\{1\} \varsubsetneqq K(s, \rho) \subset T \text { when } \alpha=0, \\
(27) \quad K(\sigma, R)=K(s, \rho) \subset T \quad \text { when } \alpha=1, \\
(28) \quad K(\sigma, R)=K(s, \rho) \subset T \quad \text { when }|\alpha|=1, \alpha \neq 1 \text { for } s=1, \\
(29) \quad K(\sigma, R) \varsubsetneqq K(s, \rho) \subset T \quad \text { when } \alpha \in D_{1},|\alpha| \neq 1, \alpha \neq 0, \\
\text { (30) } \quad K(s, \rho) \varsubsetneqq K(\sigma, R) \subset T \quad \text { when } \alpha \in D_{2} \cap D_{3},|\alpha| \neq 1, \\
\text { (31) } K(s, \rho), K(\sigma, R) \subset T, \quad K(\sigma, R) \backslash K(s, \rho) \neq \emptyset, \\
K(s, \rho) \backslash K(\sigma, R) \neq \emptyset \quad \text { when } \alpha \in D_{4} \cup D_{3} .
\end{array}
$$

In the cases when $\alpha \in D_{2} \backslash D_{3}$ or $D_{4} \backslash D_{3}$, the disc $K(\sigma, R) \notin T$; $E, \sigma, R, D_{1}-D_{4}, T$ are defined by formalae (4), (12), (14), (16-19) and (24) respectively.

4 .

For obvious reasons, we will confine our attention to $\alpha \in D_{3}$, that is, to cases (26-31). Then both the discs considered are subsets of the half-plane $T$. We shall prove 
THEOREM 1. Let $(s, \rho) \in E, \alpha \in D_{3}, \quad \sigma=1-\alpha+\alpha s, R=|\alpha| \rho$. Then the operator (3) maps the class $S^{c}(s, \rho) \subset S^{c}$ onto the class $S^{c}(\sigma, R) \subset S^{c}$, where

$\begin{array}{ll}\text { (26') } S^{c}(\sigma, R)=\{z\} \varsubsetneqq S^{c}(s, \rho) \text { when } \alpha=0, \\ \text { (27') } S^{c}(\sigma, R)=S^{c}(s, \rho) & \text { when } \alpha=1, \\ \left(28^{\prime}\right) S^{c}(\sigma, R)=S^{c}(s, \rho) & \text { when }|\alpha|=1, \alpha \neq 1 \text { for } s=1, \\ \left(29^{\prime}\right) S^{c}(\sigma, R) \varsubsetneqq S^{c}(s, \rho) & \text { when } \alpha \in D_{1},|\alpha| \neq 1, \alpha \neq 0, \\ \left(30^{\prime}\right) S^{c}(s, \rho) \varsubsetneqq S^{c}(\sigma, R) & \text { when } \alpha \in D_{2} \cap D_{3},|\alpha| \neq 1,\end{array}$

(3I') $S^{c}(\sigma, R) \backslash S^{c}(s, \rho) \neq \varnothing, \quad S^{c}(s, \rho) \backslash S^{c}(\sigma, R) \neq \emptyset$ when $\alpha \in D_{4} \cup D_{3}$.

Proof. Let $f \in S^{c}(s, p)$ and $E=I(f)$. Then from (9) and (3) we get (11) and, in consequence,

$$
\left|1+\left(z F^{\prime \prime}(z) / F^{\prime}(z)\right)-\sigma\right|=|\alpha|\left|1+\left(z f^{\prime \prime}(z) / f^{\prime}(z)\right)-s\right|, z \in \cdot K .
$$

So, from (5) and (14) we obtain (13), and, thus, $F \in S^{c}(\sigma, R)$. Since $\alpha \in D_{3}$, we infer from (24) that $F \in S^{c}$. Of course, $f \in S^{c}$ by the definitions of the class $S^{c}(s, \rho)$ and the set $E$. In view of (10) and (32), the converse is true, too. It remains to prove relations $\left(26^{\prime}-31^{\prime}\right)$.

So, if $\alpha=0$, then to each function $f \in S^{c}(s, \rho)$ there corresponds a function $F_{0}(z) \equiv z$. Consequently, the class $S^{c}(\sigma, R)$ reduces to the function $F_{0}$ which is, of course, one of the functions of the family $S^{c}(s, \rho)$

If $\alpha=I$, then $F=I(f)=f$, whence we get $\left(27^{\prime}\right)$.

In case (28') we have $\sigma=s=1, R=\rho$, and thus, $\pi(\sigma, R)=K(s, \rho)$. Hence the classes are equal. Obviously, here we do not have to deal with the identity mapping, as in (27').

If $\alpha \in D_{1},|\alpha| \neq 1, \alpha \neq 0$, then from (29) we infer that (29') 
holds. Analogously, from (390) and (31) we get (30') and (31'), respectively.

Note that the solution $f^{*}(z)=z+\ldots$ of the differential equation (see (8))

$$
1+z f^{\prime \prime}(z) / f^{\prime}(z)=h(z), z \in K,
$$

where the function $h$ is defined by formulae (6) and (7), has the form

$$
f^{*}(z)= \begin{cases}\int_{0}^{z}(1+b u)^{(a-b) b^{-1}} d u & \text { for } s \neq 1, \\ \int_{0}^{z} e^{\rho u} d u & \text { for } s=1 .\end{cases}
$$

Let $F^{*}=I\left(f^{*}\right)$. Then from (3) and (34) we have

$$
F^{*}(z)= \begin{cases}\int_{0}^{z}(1+b u)^{\alpha(a-b) b^{-1}} d u & \text { for } s \neq 1, \\ \int_{0}^{z} e^{p \alpha u} d u & \text { for } s=1 .\end{cases}
$$

If $s \neq 1$, we then obtain

$$
1+z F^{* \prime \prime}(z) / F^{* \prime}(z)=\frac{1+(b+\alpha(a-b)) z}{1+b z}, z \in K .
$$

Since the homography occurring on the right-hand side of formula (35) maps the disc $K$ onto the disc with centre at $\sigma=1-\alpha+\alpha$ and radius $R=|\alpha| \rho$, therefore the results obtained in Theorem 1 cannot be improved.

Analogously, when $s=1$, we get

$$
1+z F^{* \prime \prime}(z) / F^{* \prime}(z)=1+\alpha \rho z,
$$

whence $\sigma=1, R=|\alpha| \cdot \rho$; so, also in this case, the results of Theorem 1 are unimprovable.

From the main theorem we obtain

COROLLARY 1 [10]. If $f \in S^{\mathcal{C}}(s, \rho), s=\bar{s}, \alpha=\bar{\alpha}$, then the function $F=I(f) \in S^{c}(s, p)$ if and only if $-\tilde{\alpha} \leq \alpha \leq 1$ where 


$$
\tilde{\alpha}= \begin{cases}\frac{\rho-(s-1)}{\rho+(s-1)} & \text { when } s>1, \\ 1 & \text { when } s=1, \\ \frac{\rho+(s-1)}{\rho-(s-1)} & \text { when } \frac{1}{2}<s<1 .\end{cases}
$$

In deed, the intersection of the set $D_{1}$ with the real axis is the interval $\left\langle\alpha_{1}, 1\right\rangle$ where $\alpha_{1}=-(\rho-|s-1|)(\rho+|s-1|)^{-1}$, whence we immediately obtain formulae (36).

COROLLARY 2. If $f \in S^{c}(s, \rho), s=\bar{s}$, then the function $\vec{r}=I(f) \in S^{c}(s, \rho)$ for $\alpha \in K\left(0,-\alpha_{1}\right)$.

This follows from the fact that min $r_{1}(t)=-a_{1}=r_{1}(\pi)$ where $r=r_{1}(t)$ is the equation of the boundary of the set $D_{1}$. In paper [13], in the case under consideration, the disc $K\left(0, \frac{1}{2} \rho^{-1}(\rho-s+1)\right)$ with radius less than $-\alpha_{1}$ was obtained. So the result from [13] in question is not sharp.

COROLLARY 3 [8]. If $f \in S^{c}$, then the function $F=I(f) \in S^{c}$ for each $\alpha \in\langle 0,1\rangle$. Moreover, the extremal function is of the form $f^{*}(z)=\int_{0}^{z}(1+u)^{-2} d u$.

This corollary follows directly from (36), (35) and (34). One should put $s=\rho$ and pass to the limit with $\rho \rightarrow+\infty$.

In the case $s=\bar{s}$ and $\alpha=\bar{\alpha}$, from Theorem 1 one can, of course, also obtain more specific properties of the operator (3). For instance, if $s \neq 1$ and $\alpha \in\left(1, \alpha_{2}\right)$, then from (30') we deduce that, for each function $f \in S^{c}(s, \rho), F=I(f) \in S^{c}(\sigma, R)$ with $S^{c}(s, \rho) \underset{\ddagger}{\subsetneq} S^{c}(\sigma, R) \subset S^{c}$. For $\alpha>\alpha_{2}, F=I(f)$ does not have to be a convex univalent function. 


\section{5.}

Let $S_{\beta}^{c}, 0 \leq \beta<1$ (see [6]), stand for the family of functions of form (1) satisfying the condition

$$
\operatorname{Re}\left[\bar{I}+\left(z f^{\prime \prime}(z) / f^{\prime}(z)\right)\right]>\beta, z \in K .
$$

Let $\alpha \geq 0, f \in S_{\beta}^{c}$. Then from (3) and (9) we get

$\operatorname{Re}\left[1+\left(z F^{\prime \prime}(z) / F^{\prime}(z)\right)\right]=\alpha \operatorname{Re}\left[1+\left(z f^{\prime \prime}(z) / f^{\prime}(z)\right)\right]-\alpha+1 \geq \alpha \beta-\alpha+1$, $z \in K$.

Consequently, from (37) and (33) we have

THEOREM 2. Let $0 \leq \beta<1,0 \leq \alpha \leq(1-\beta)^{-1}$. Then operator (3) maps the class $S_{\beta}^{c}$ onto the class $S_{\delta}^{c}$ where $\delta=\alpha \beta-\alpha+1$, where

$$
\begin{aligned}
& S_{\delta}^{c} \subsetneq S_{\beta}^{c} \text { when } 0 \leq \alpha<1, \\
& S_{\delta}^{c}=S_{\beta}^{c} \text { when } \alpha=1, \\
& S_{\delta \supsetneqq}^{c} S_{\beta}^{c} \text { when } 1<\alpha \leq(1-\beta)^{-1} .
\end{aligned}
$$

The extremaz function is of the form $f^{*}(z)=\int_{0}^{z}(1+u)^{2(\beta-1)} d u$.

Relations (38-40) constitute another type of generalizations of the result of [8].

\section{6.}

Let $S_{\beta}^{v}, 0<\beta \leq \pi / 2$, denote the family of functions of the form (1) satisfying the condition

$$
\left|\arg f^{\prime}(z)\right|<\beta, z \in K, \quad \arg 1=0 .
$$

Proceeding analogously, we obtain, from (10),

THEOREM 3. Let $0<\beta \leq \frac{1}{2} \pi,-1 \leq \alpha \leq 1$. Then operator (3) maps the class $S_{\beta}^{v}$ onto the class $S_{|\alpha| \beta}^{v} \subset S_{\beta}^{v}$. The extremal function is of 
the form $f^{*}(z)=\int_{0}^{z}[(1-u) /(1+u)]^{\beta} d u$

Indeed, as is known, functions of the class $s_{\pi / 2}^{v}[12]$ are univalent; thus, for $f \in S_{\beta}^{\mathcal{V}}$, we have $f^{\prime}(z) \neq 0$ for $z \in K$. Besides, by $(10), \arg F^{\prime}(z)=\alpha \arg f^{\prime}(z)$, whence, in view of (41), we obtain the assertion of the theorem.

\section{7.}

Let us take the functions

$$
g(z)=\sum_{k=0}^{\infty} g_{k} z^{k}, \tilde{g}(z)=\sum_{k=0}^{\infty} \tilde{g}_{k} z^{k},
$$

holomorphic in the disc $K, g_{0}=\tilde{g}_{0} \neq 0$, and satisfying in $K$ the condition

$$
g(z) / \tilde{g}(z) \prec h(z)
$$

where $h$ is of form $(6)$ and $(7),(s, p) \in E$. Then, proceeding as in paper [4], we obtain the following theorem on coefficients.

THEOREM 4. For each $(s, \rho) \in E$ and any pair of functions $g, \tilde{g}$ satisfying conditions (42) and (43), the following estimations

$$
\begin{aligned}
& \left|g_{1}-\tilde{g}_{1}\right| \leq|a-b|=\left(\rho^{2}-|s-1|^{2}\right) \rho^{-1}, \\
& \left|g_{n}-\tilde{g}_{n}\right|^{2} \leq|a-b|^{2}\left|g_{0}\right|^{2}-\sum_{k=1}^{n-1} A_{k}, n=2,3, \ldots,
\end{aligned}
$$

$$
\sum_{k=1}^{\infty} A_{k} \leq|a-b|^{2}\left|g_{0}\right|^{2}
$$

take place, where

$$
A_{k}=\left|g_{k}-\tilde{g}_{k}\right|^{2}-\left|a \tilde{g}_{k}-b g_{k}\right|^{2}, k=1,2, \ldots
$$

From (44), (45) and (47) we get

COROLLARY 4. If, for fixed $g, \tilde{g},(s, \rho) \in E$, all numbers $A_{k}$ are non-negative, then 


$$
\left|g_{n}-\tilde{g}_{n}\right| \leq\left(\rho^{2}-|s-1|^{2}\right) \rho^{-1}, \quad n=1,2, \ldots,
$$

for a given $n$, equality holds only when $A_{k}=0$ for $k=1, \ldots, n-1$.

COROLLARY 5. If, for fixed $g, \tilde{g},(s, \rho) \in E$, there exists an index $N(N=3,4, \ldots)$ such that $A_{k} \leq 0$ for $k=1,2, \ldots, N-2$ and $A_{k} \geq 0$ for $k=N-1, N, \ldots$, then

$$
\left|g_{n-1}-\tilde{g}_{n-1}\right|^{2} \leq \begin{cases}|a-b|^{2}\left|g_{0}\right|^{2}, & n=2, \\ |a-b|^{2}\left|g_{0}\right|^{2}-\sum_{k=1}^{n-2} A_{k}, & n=3, \ldots, N, \\ |a-b|^{2}\left|g_{0}\right|^{2}-\sum_{k=1}^{N-2} A_{k}, & n=N+1, N+2, \ldots .\end{cases}
$$

Let $f$ of form (1) belong to $s^{c}(s, \rho),(s, \rho) \in E$. Put $g(z) \equiv f^{\prime}(z)+z f^{\prime \prime}(z), \tilde{g}(z) \equiv f^{\prime}(z)$. Then from (6) to (8), (42), (43), (46), (48) and (49) we obtain the estimations of coefficients of functions in the classes $S^{c}(s, \rho)$. We have

THEOREM 5. Let $f$ of form (1) be any function of the class $S^{c}(s, \rho),(s, \rho) \in E . \quad$ If $(s, \rho) \in C_{1}$, then

$$
\left|a_{n}\right| \leq n^{-1}(n-1)^{-1} A, n=2,3, \ldots .
$$

If $(s, \rho) \in C_{2}$, then

$$
\left|a_{n}\right| \leq \frac{1}{n !} \prod_{k=0}^{n-2}\left|e^{i \gamma_{A-k b}}\right|, n=2,3, \ldots, N,
$$

and

$$
\left|a_{n}\right| \leq \frac{1}{(N-2) ! n(n-1)} \prod_{k=0}^{N-2}\left|e^{i \gamma_{A-k b}}\right|, n=N+1, N+2, \ldots,
$$

where

$$
\begin{aligned}
& N=\left[k_{0}\right]+2, \quad k_{0}=\left(\rho^{2}-\operatorname{Im}^{2} s\right)^{\frac{1}{2}}+\operatorname{Re} s-1>1, \\
& A=\rho^{-1}\left(\rho^{2}-|s-1|^{2}\right), \quad b=\rho^{-1}|s-1|,
\end{aligned}
$$




$$
\begin{aligned}
& C_{1}=\{(s, \rho) \in E: \operatorname{Re} s<2 \text { and } \rho \leq|s-2|\} \\
& C_{2}=\{(s, \rho) \in E: \operatorname{Re} s \geq 2 \text { or }|s-2|<\rho\} .
\end{aligned}
$$

Besides, we have

$$
\sum_{k=1}^{\infty}(k+1)\left|a_{k+1}\right|^{2}\left|k^{2}-\right| A e^{i \gamma_{-}}-\left.b k\right|^{2} \mid \leq A^{2} .
$$

Estimations (50) and (51) are sharp.

We omit the proof of the above theorem (see, for example [4]). Of course, in the case when $\rho=s \rightarrow+\infty$, from (51) we obtain the well-known estimations of coefficients in the full family $s^{c}\left(\left|a_{n}\right| \leq 1, n=2,3, \ldots\right)$.

\section{References}

[1] Z.J. Jakubowski, "On the coefficients of Carathéodory functions", Bull. Acad. Polon. Sci. Sér. Sci. Math. 19 (1971), 805-809.

[2] Z.J. Jakubowski, "On the coefficients of starlike functions of some classes", Buzz. Acad. Polon. Sci. Sér. Sci. Math. 19 (1971), $811-815$.

[3] Z.J. Jakubowski, "On some applications of the Clunie method", Ann. Polon. Math. 26 (1972), 211-217.

[4] Z.J. Jakubowski, "On the coefficients of starlike functions of some classes", Ann. Polon. Math. 26 (1972), 305-313.

[5] Z.J. Jakubowski, "On some properties of extremal functions of Carathéodory", Ann. Soc. Math. Polon. Ser. I 17 (1973), 71-80.

[6] Z.J. Jakubowski and J. Kamiński, "On some properties of MocanuJanowski functions", Rev. Roumaine Math. Pures Appl. 23 (1978), 1523-1532.

[7] W. Janowski, "Extremal problems for a family of functions with positive real part and some related families", Bull. Acad. Polon. Sci. Sér. Sci. Math. 17 (1969), 633-637. 
[8] Y.J. Kim and E.P. Merkes, "On certain convex sets in the space of locally schlicht functions", Trans. Amer. Math. Soc. 196 (1974), $217-224$.

[9] V. Kumar and S.L. Shukla, "Jakubowski starlike integral operators", J. Austral. Math. Soc. Ser. A 36 (1984), 117-127.

[10] V. Kumar and S.L. Shukla, "Jakubowski convex integral operators", $J$. Austral. Math. Sce. Ser. A (to appear).

[11] R.J. Libera and A.E. Livingston, "Bounded functions with positive real part", Caechoslovak Math. J. 22 (97) (1972), 195-207.

[12] K. Noshiro, "On the theory of schlicht functions", J. Fac. Sci. Hokkaido Univ. Ser. I $2(1934 / 35), 129-155$.

[13] R.K. Pandey and G.P. Bhargava, "On convex and starlike univalent functions", Bull. Austral. Math. Soc. 28 (1983), 393-400.

Institute of Mathematics,

Lódź University,

ul. Stefana Banacha 22,

90-238, Lódź,

Poland. 